\title{
The eyes have it!: An fMRI investigation
}

\author{
Alan Kingstone, ${ }^{\mathrm{a}, *}$ Christine Tipper, ${ }^{\mathrm{a}}$ Jelena Ristic, ${ }^{\mathrm{a}}$ and Elton Ngan ${ }^{\mathrm{b}}$ \\ a Department of Psychology, University of British Columbia, Vancouver, BC, Canada V6T 1Z4 \\ ${ }^{\mathrm{b}}$ Department of Psychiatry, University of British Columbia, Vancouver, BC, Canada V6T $1 Z 4$
}

Accepted 12 February 2004

Available online 9 April 2004

\begin{abstract}
For the past several years it has been thought that cues, such as eye direction, can trigger reflexive shifts in attention because of their biological relevance and their specialized neural architecture. However, very recently, Ristic, Friesen, and Kingstone (2002) reported that other stimuli, such as arrows, trigger reflexive shifts in attention in a manner that is behaviourally identical to those triggered by eyes. Nevertheless these authors speculated that reflexive orienting to gaze direction may be subserved by a neural system - the superior temporal sulcus (STS) - that is specialized for processing eyes. The present study presents fMRI data that provide direct and compelling empirical support to this proposal. Subjects were presented with fixation stimuli that, based on instruction, could be perceived as eyes or as another type of directional cue. Both produced equivalent shifts in reflexive attention, replicating Ristic et al. However, the neural systems subserving the two forms of orienting were not equivalent-with the STS being engaged exceptionally when the fixation stimulus was perceived as eyes.
\end{abstract}

(C) 2004 Elsevier Inc. All rights reserved.

\section{Introduction}

Friesen and Kingstone (1998) first reported that humans attend reflexively to locations and objects that are being looked at by other people. The fact that eye direction is of such fundamental importance to human behaviour is underscored by a wealth of research indicating that a particular region of the human brain-the superior temporal sulcus (STS) - is specialized from birth to give preference to processing the eyes of others. Indeed, deficiencies in the STS may be catastrophic, leading to complex disabilities such as autism (see Kingstone, Friesen, \& Gazzaniga, 2000 for a review).

Recently, however, Ristic, Friesen, and Kingstone (2002) discovered that eyes are not unique in their ability to trigger a reflexive shift in attention based on symbolic directional information. Arrows produced a reflexive attentional shift in healthy children and adults that was indistinguishable from that produced by eyes. Does this mean that the same neural systems subserve reflexive orienting in response to eyes and arrows? Based on split-

\footnotetext{
${ }^{*}$ Corresponding author.

E-mail address: alan.kingstone@ubc.ca (A. Kingstone).
}

brain data, Ristic et al. argued that the two systems are different. They noted that where reflexive orienting to gaze direction was lateralized to face processing mechanisms normally specific to the right hemisphere, reflexive orienting to arrow direction was present in both hemispheres. The different patterns of lateralization observed for eyes and arrows with split-brain patients, however, may have been due to the fact that testing was conducted at different times and with different stimuli, rather than due to eyes and arrows being subserved by different neural systems.

The present study addressed this issue directly while avoiding these previous shortcomings. Functional magnetic resonance imaging (fMRI) was performed while healthy adults responded to a target that appeared to the left or right of central fixation. The fixated stimulus was ambiguous in that it could be perceived either as eyes looking left or right, or as a car. The critical manipulation was whether subjects were informed that the stimulus was a face or a vehicle (Ristic \& Kingstone, 2002; see Fig. 1A). Based on the Ristic et al. split-brain data, we reasoned that a reflexive attentional shift could be triggered by the symbolic cue regardless of whether it was seen as eyes or a car. The key question was whether, as Ristic et al. 
A

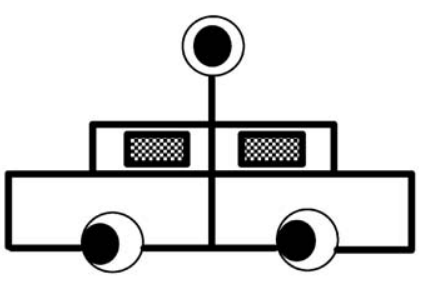

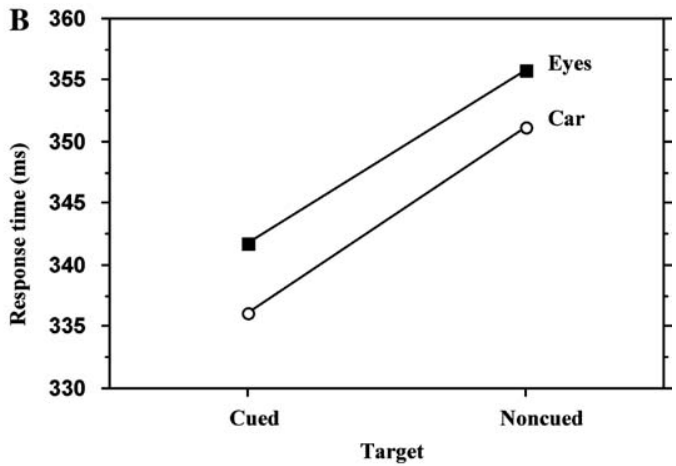

Target

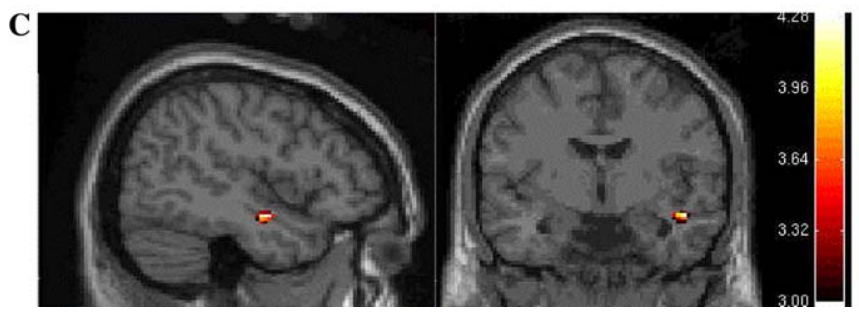

Fig. 1. (A) Illustrates the fixation stimulus used in the present study, which can be perceived as a car or as a hat pulled down to the eyes. For instance, if perceived as eyes, the target is appearing at the gazed-at (cued) location. (B) The correct RT for a target appearing at the cued and noncued locations when the fixation stimulus is perceived as Eyes or a Car. Note that there is a reliable effect of cue for both Eyes and Car, indicating that attention was shifted to the cued location for both cue stimuli. Because the cue did not predict where a target would appear (e.g., the target was as likely to appear at the gazed-at location as the nongazed-at location), this cuing effect is attributed to a reflexive shift in visuospatial attention. (C) Sagittal and coronal views of the statistical parametric map, indicating where the BOLD response was greater for the Eyes condition than for the Car condition. This contrast revealed a unique area of activation specific to the Eyes condition in the superior temporal sulcus of the right hemisphere.

predicted, the right hemisphere STS would be preferentially activated when the fixation stimulus was perceived as eyes.

\section{Methods}

\subsection{Subjects}

Twenty-two participants (12 male, mean age $=31$ years) were recruited through advertisement, and were either paid $\$ 10$ or given an MRI film of their brain to participate in the study. All participants were right handed. Pre-screening was conducted to rule out psychological and neurological disorders, as well as MRI contraindications.

\subsection{Stimuli}

Stimuli were presented in an event-related fMRI design. Each trial consisted of the presentation of the eyes/ car stimulus (Fig. 1A) at fixation, followed $600 \mathrm{~ms}$ later by an asterisk target positioned approximately $10^{\circ}$ to the left or right of centre. The target remained on the screen for $750 \mathrm{~ms}$. Participants were required to press a button as soon as they detected the target. Between each trial, a fixation cross was presented for either 6,7 , or $8 \mathrm{~s}$. Trials were presented in 9-min scanning runs, consisting of 30 cued and 30 noncued trials. Cued and noncued trials were initially randomized throughout one run, and this same run was always presented.

\subsection{Method}

Functional MRI data were collected on a clinical GE 1.5T system equipped with a Horizon Echo-Speed upgrade. Functional volumes were collected with a gradient echo pulse sequence $(\mathrm{TR}=3000 \mathrm{~ms}, \mathrm{TE}=40 \mathrm{~ms}$, $90^{\circ}$ flip angle, FOV $24 \times 24 \mathrm{~cm}, 64 \times 64$ matrix, $62.5 \mathrm{kHz}$ bandwidth, $3.75 \times 3.75$ in-plane resolution, $5.00 \mathrm{~mm}$ slice thickness, 29 slices), sensitive to blood oxygen level dependent (BOLD) contrast. Stimuli were projected from an LCD projector onto a screen mounted at the foot of the bore. An angled mirror reflected images on the screen to the participants' field of view.

Participants took part in either two or three scanning runs. The perception of the ambiguous eyes/car stimulus was manipulated with instructions that differed between runs. For participants who completed two scanning runs, the instruction for the first run was to view the stimulus as a car. The instruction for the second run was to view the stimulus as eyes and a hat. For participants who completed three scanning runs, the instruction for the first two runs was to view the stimulus as a car, while the instruction for the third run was to view the stimulus as eyes and a hat. Three scanning runs were used with 10 
of the 22 participants in order to account for the effect of practice, as counterbalancing was not possible due to the nature of the ambiguous stimulus. Once the stimulus is seen as eyes, it is very difficult to see it as a car (Ristic $\&$ Kingstone, 2002). For participants who completed three runs, the two Car conditions (which did not differ $F<1$ ) were averaged and submitted to further analysis.

\section{Results}

\subsection{Behavioural data}

Fig. 1B shows that response time (RT) was faster when a target appeared at a cued location than a noncued location. Consistent with Ristic et al. (2002), this occurred regardless of whether the directional cue was perceived as a pair of eyes or as a car. It is important to note that these cuing effects reflect shifts in reflexive attention, as opposed to voluntary attention, because the cue did not predict where the target would occur.

A repeated-measures ANOVA of the correct RTs, with target location (cued/noncued) and stimulus context (eyes/car) as factors confirmed that there was a significant cuing effect, $F(1,21)=41.42, p<.0001$, that did not vary as a function of stimulus context $(F \mathrm{~s}<1$ for both a main effect and interaction). Order had no effect on cueing $(F<1)$. Errors occurred on well under $5 \%$ of the trials and did not conflict with the latency data. In summary, the behavioural data agree with our previous observations that directional cues, such as eyes and arrows, produce reflexive shifts of attention to the cued location that are behaviourally equivalent.

\subsection{Imaging data}

Although the behavioural effects of reflexive orienting are equivalent for eyes and cars, the question of whether they activate the same neural architecture remains open. Functional MRI data were processed and modeled, and statistical procedures were conducted using SPM99. A paired $T$ test was conducted at each voxel (unit of resolution), comparing the BOLD responses in the Eyes and Car conditions. Fig. $1 \mathrm{C}$ shows a map of regions where the difference yielded a $T$ statistic of 3.0 or higher $(p \lesssim .003$, uncorrected). This map indicates that one area-the superior temporal sulcus (STS) - was uniquely engaged when orienting to a stimulus that was perceived as eyes.

\section{Discussion}

Ristic et al. (2002) speculated that although equivalent shifts in reflexive attention can be triggered by different directional stimuli (e.g., eyes, arrows, car) they may not be subserved by the same neural architecture. Furthermore, they hypothesized that orienting to eyes may activate the right hemisphere STS, as previous studies suggested that this region may be specialized for processing gaze information.

The present results support this hypothesis. Subjects were presented with a stimulus that could be perceived as eyes or as a car (Ristic \& Kingstone, 2002). Both percepts produced equivalent reflexive shifts in attention to the cued location when measured behaviourally. The STS, however, was uniquely engaged when the stimulus was perceived as eyes. As such, the present data converge with, and support, the conclusions presented by Ristic et al. (2002). Here the authors argued that behavioural equivalence does not mean that the underlying neural architecture is engaged equivalently. In the present study we find that reflexive orienting to eye direction is subserved by a neural architecture-STS - that appears to be engaged especially when the stimuli are represented as eyes.

\section{Acknowledgments}

This research was supported by a grants to AK from the Human Frontier Science Program, the Natural Sciences and Engineering Research Council of Canada, and the Michael Smith Foundation for Health Research.

\section{References}

Friesen, C. K., \& Kingstone, A. (1998). The eyes have it!: Reflexive orienting is triggered by nonpredictive gaze. Psychonomic Bulletin \& Review, 5, 490-495.

Kingstone, A., Friesen, C. K., \& Gazzaniga, M. S. (2000). Reflexive joint attention depends on lateralized cortical connections. Psychological Science, 11, 159-166.

Ristic, J., Friesen, C. K., \& Kingstone, A. (2002). Are eyes special? It depends on how you look at it. Psychonomic Bulletin \& Review, 9 , 507-513.

Ristic, J., \& Kingstone, A., (2002). Is it a car or a hat? Automaticity of orienting to gaze direction. Paper presented to the Canadian Society of Brain, Behaviour and Cognitive Science. 